\title{
EML BASED URBAN FIRE INCIDENT MODELING METHOD AND PROTOTYPE
}

\author{
An $\mathrm{He}^{1}$, Wei Wang ${ }^{1,2}$, Wenying $\mathrm{Du}^{1}$, Chao Wang ${ }^{1, *}$, Nengcheng Chen ${ }^{1,2}$ \\ ${ }^{1}$ State Key Laboratory of Information Engineering in Surveying, Mapping and Remote Sensing, Wuhan University, Luoyu Road No. \\ 129, Wuhan 430079, China - (an1125 @whu.edu.cn, wangwei8091@163.com, duwenying, c.wang, cnc@whu.edu.cn) \\ ${ }^{2}$ Collaborative Innovation Center of Geospatial Technology, Wuhan, China
}

\section{Commission II, WG II/5}

KEY WORDS: urban fire disaster, Event pattern Markup Language, MetaObject Facility, IOT, Dynamic modeling

\begin{abstract}
:
Urban fire disasters are one of the most frequent disasters that threaten public safety and social development in cities. Most urban fire disasters feature dynamics and processes. The locations, time, meteorological conditions and surroundings of each fire incidents are diverse, besides the traffic conditions around the fire place would be changing dynamically. At present, there is no unified modeling language to describe urban fire incidents, which makes it difficult to obtain experience from historical cases and hard to describe. This paper used the Event pattern Markup Language (EML) to model urban fire incidents from an observation perspective based on MetaObject Facility (MOF) framework, designed and implemented an urban fire incident modeling prototype. It can realize the information description of urban fire disasters, and provide descriptions of different observation missions during the process of the fire incidents. The detailed description of the process allows multiple observations based on the expression of EML to be performed, and offer evidence for emergency response phase and post-mortem archiving. The real data of the "12.1" major fire in Tianjin, China in 2017 was used as a use-case to show the modeling results of the entire fire incident in four phases-- mitigation, preparedness, response, and recovery. The results show that this modeling method proposed in this paper can represent the dynamic information of urban fire incidents and achieve the dynamic modeling of urban fire incidents.
\end{abstract}

\section{INTRODUCTION}

\subsection{Background}

According to the data released by the National Data Network of China, from 2006 to 2012, the number of fires and direct economic losses generally showed an upward trend. Ninety-nine percent of these fires were general fires, with an average annual direct economic loss of up to 1.65 trillion CNY. The average number of deaths due to fire disasters is 1,319 for each year (China Statistics, 2019). Therefore, for most cities in China, the risk of fire has an overall increase trend in the future. This is an arduous task for maintaining social harmony, stability and safeguarding people's property and life. The premise and foundation of disaster prevention, mitigation and rescue is to uniformly express and manage the information of urban fire incidents, and utilize observation data to model the dynamic process. According to the Federal Emergency Management Agency (FEMA), emergency management is divided into four phases: disaster mitigation, preparedness, emergency response, and recovery, and the whole process is dynamic (Haddow, 2003). However, China currently adopts the "one case, three systems" emergency management system. "One case" refers to emergency plans, and "three systems" is the abbreviation of emergency system, emergency mechanism, and related legal systems (Zhong, 2009). In spite of the truth is that China's emergency management has developed rapidly in recent years, there are still many shortcomings because of its late stars, and there is a lack of a unified management mechanism among various departments, different disasters are managed by different departments, and the links between different departments are not close enough. A unified and standardized model of disasters is significant to exchanging the necessary information during urban fire disasters, which should be using a simple and useful language to express the occurrence and development process of fire incident. So that the information exchange during the emergency management process can be more convenient, and the efficiency of government emergency management can be improved.

\subsection{Research Status}

At present, many scholars have proposed many different methods in modeling fire incidents. There are urban fire emergency rescue process models based on petri nets, which respectively model the docking police process, expert decisionmaking process, emergency rescue disposal, and subsequent disposal processes (Liu, 2006). There is also a model based on Petri nets combined with GIS, and the GIS was used to analyse spatial information based on Petri nets to develop a model for urban fire rescue and dispatching (Zhu, 2005). Other scholars have proposed a building fire emergency decision model based on CBR and Bayesian networks. Bayesian networks was used to extract key attributes of bazaar building fires for retrieval, and select cases with large similarities as matching cases, provide decision-making for emergency rescue (Xie, 2018). There are many other modeling methods, however, most of them are static expressions, and they cannot be combined with real-time data to promote the evolution of incidents. Besides, their descriptions of fire incidents are too simple, and the rules of incidents development and the evolution of characteristic processes are not detailed and clear.

In view of the above shortcomings, an urban fire modeling method based on the Event pattern Markup Language (EML) and MetaObject facility (MOF) framework was proposed in the paper, it can provide detailed information description of the entire fire process, and dynamically model according to 
different observation tasks from four phases. EML was proposed by the Open Geographic Information Consortium (OGC) in 2008 and provides a description of the event mode. It consists of an event object, an event model, and event-related application capabilities. EML has four modes, the simple mode, is used to filter a single input stream, and SQL-like Where statement, there are attribute restrictions, restrictions on names, attribute values. Complex patterns link different incidents together. Timing mode, generating time events, time points match. Repeat pattern. count the number of matching subpatterns. There are also four types of selection functions used to extract information from the event set, used to select the information contained in the event, and used to extract information from the event content, which can be customized by the user (Everding, 2008).

The MOF framework is a set of modeling frameworks defined by the object management organization. It defines the modeling steps and abstract content and embodies in specific areas (OMG, 2014). MOF is proposed by the object management organization. It is an international standard: ISO / IEC 19502: Information Technology-Meta Object Facility (International standard, 2005), which uses a unified abstract syntax and hierarchical meta-level structure to describe modeling concepts of models, elements and their relationships. MOF is divided into four layers of modeling structure, which are instance layer (M0), model layer (M1), meta model layer (M2) and meta-meta model layer (M3). Combining the above characteristics of the MOF framework and the EML modeling language, a model can be established from the perspective of observation. Different observation tasks in four phases can make the model easy to understand, make the modules clear, same as the tasks and functions. By using non-dynamic specific information to describe components, makes the process of model establishment and the information expressed dynamically, and the observation data would be update in the model of a fire incident.

\section{MODELING METHOD OF URBAN FIRE INCIDENT}

\subsection{Modeling Framework}

The four-layer standard MOF architecture of urban fire incidents describes the modeling concept of the fire event metamodel and the relationships between the concepts, each layer is an example of the previous layer, and the abstraction of the next layer. The urban fire model based on MOF framework was shown in Figure 1.

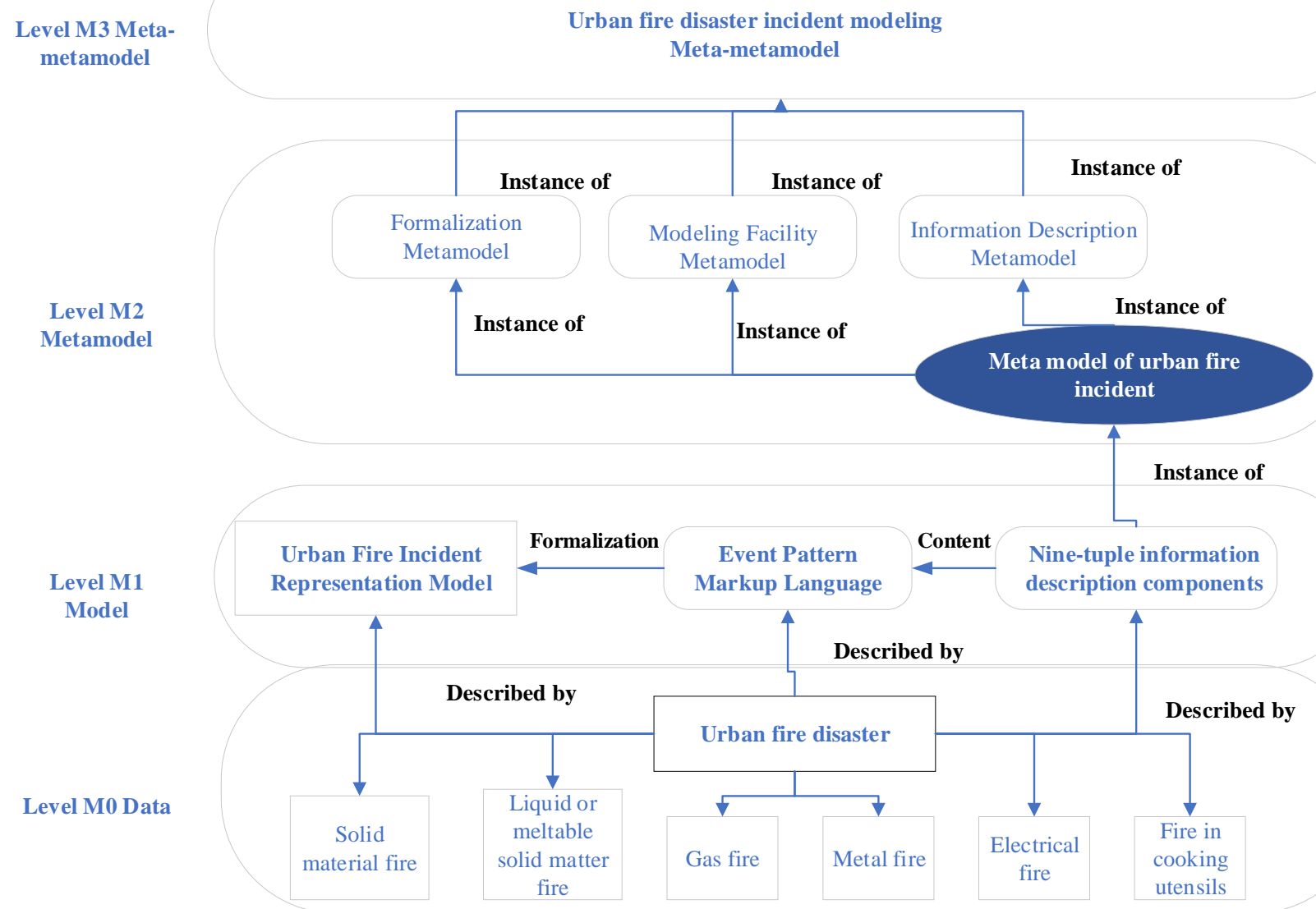

Figure 1. The urban fire incidents model based on the MOF framework

Level M3 is a meta-metamodel layer, which defines the basic concepts and relationships of the fire incident information model. It uses the metamodel as an example of a high-level abstraction and defines multiple metamodels. Level M2 is a metamodel layer, including a formalization metamodel, a modeling facility metamodel, and an information description metamodel. These three metamodels are instantiation of urban fire incident modeling meta-metamodel, and they are abstract representation of urban fire incident representation model, EML and nine-tuple information description components. The metamodel of urban fire events in the M2 layer is divided into four parts, self-description, space-time, observation and management. The self-description is made of identification and classification, which respectively indicate the name and category of the fire. Space-time refers to time and location. Observation refers to the different observation missions at four 
phases, and the process of detecting the occurrence and dynamic changing of fire incidents based on the Internet of Things data. Management includes archive, contact and service. Archive indicates all the observation information of the fire incident. Contact is the name, address, and contact information of the person who report the fire incident. Services includes service type, name, and address. Level M1 is an urban fire incident model layer, based on MOF meta-level framework, this layer consists of urban fire incident representation model, EML and nine-tuple information description components. It is an instantiation of urban fire incident meta model. The representation model of urban fire incident is composed of ninetuple information description components described by EML. The nine-tuple description information in the level M1 includes identification information, classification information, archive information, status information, time information, space information, observation information, contact information, and service information. Level M0 is real word information layer, and it consists of different categories of urban fire incidents. There are six kind of fire disasters, solid material fire, liquid fire, gas fire, metal fire, electrical fire, and fire in cooking utensils.

In the M1 layer, the urban fire representation model contains nine types of description information, as shown in the Figure 2 below. Each type of description information has different elements and different functions. These nine sets of description information form an urban fire incident meta-model.

1) Identification information: It is the identification of the typical characteristics of a fire incident. This information can correspond to a unique instance of the fire information model. Therefore, it will have a unique number and name. The name element will combine with the date, location, and fire type.
2) Classified information: It mainly expresses the type, urgency, and severity of fire incidents.

3) Time information: It covers the entire process from the occurrence to the end of the fire incident, and is expressed in $\mathrm{URC}+8$-time format.

4) Spatial information: refers to the spatial extent of the fire incident. There are two ways of expression, one is to express by location, the public uploads information, and the other is to express by latitude and longitude coordinates, which is convenient for quick display and overlay analysis on the map.

5) Mission information: During a fire incident, the observation tasks and observation data are different at different phases, and the observation methods are different. As shown in the previous table 1 , if the mission is completed by ground data, it will directly access the ground data. If the mission is observed by sensors, it will directly access the sensor data. Observation tasks will change according to time and phases of incidents.

6) Status information: Use the observation information obtained during the preparedness phase to determine the fire occurrence status, and then use the position of the alarm sensor to determine the possible fire area and location.

7) Archive information: It includes the observation information of different phases of the fire, the place of occurrence, the event, the category, etc., which is convenient for querying historical events in the future.

8) Contact information: refers to the contact information of the event provider, including name, unit, address, telephone, etc., for subsequent management of the event. If there is any information needs to be supplemented, users can contact the publisher through the contact information.

9) Service information: including service type, name and address, mainly referring to service related to the sensors. Filter and anomaly detection through sensor incident services, and issue warning to the users of this software.

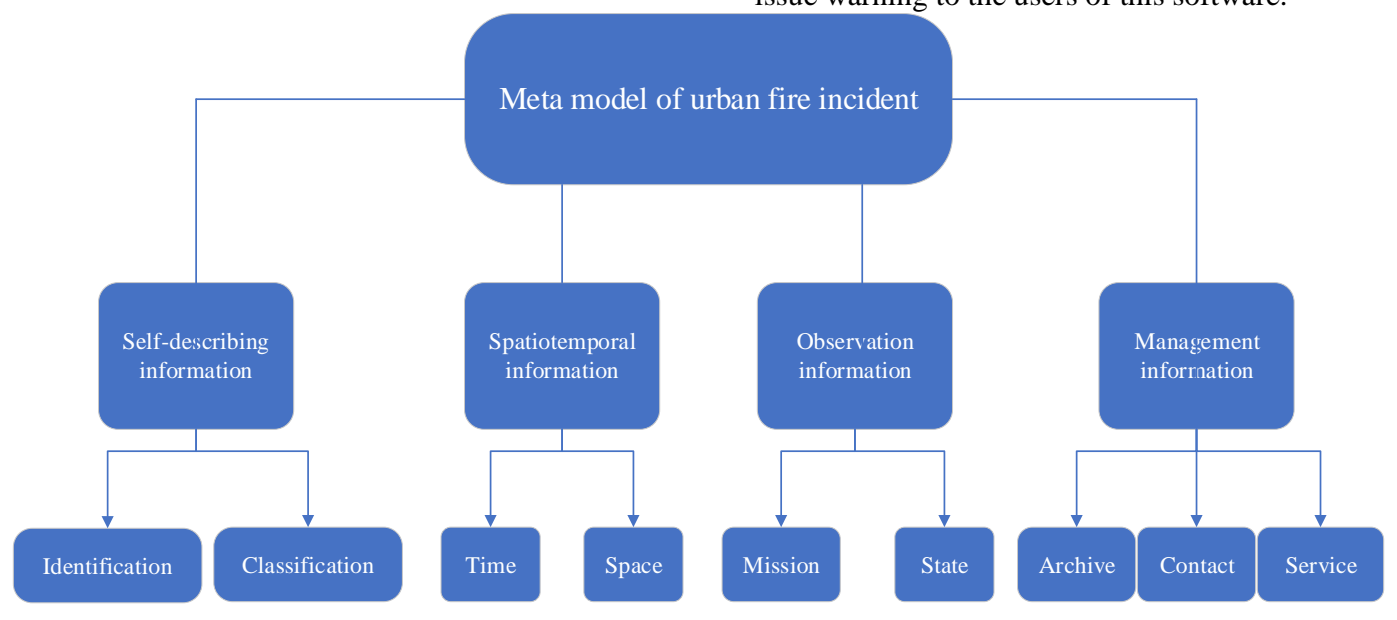

Figure 2. Meta model of urban fire incident

\subsection{Modeling Method}

Most fires are manually reported to the 199-fire alarm platform, where the staff will ask information about the location of the fire and the contact information of the person who report the fire. Subsequently, fire protection forces around the fire site were sent to there, and the surrounding road conditions were observed through Sky-net cameras to formulate the best rescue route. After rushing to the scene, the firemen will design a fire extinguishing plan based on the weather information of that day and the site condition. After the fire operation is completed, the government will collect the casualty and socioeconomic data base on manual statistic survey. The cause of the fire will be investigated after the fire, and the person who is responsible to the fire will be published. In the actual fire-fighting process, the data available from the sensors in the fire field were not used to speculate on the development of the fire. The entire fire-fighting operation was judged manually, and lack of records of real-time data in the fire will cause some unnecessary mistakes. The shortcomings in the actual rescue process are mainly the lack of data on historical events and on-site data during the fire.

To improve the deficiencies in the actual firefighting process, this paper proposal the observation mission, data and approach method in four phases. According to FEMA, the fire incident is divided into four phases, mitigation, preparedness, emergency 
response, and recovery. The observation tasks, observation methods, and purposes of each phases are different. As shown in the Table 1 , there are details of every observation mission, data and method. The disaster mitigation phase mainly collects basic data about fire incidents, including geographic basic data, location data of fire stations, hospitals, emergency resources (fire hydrants, fire extinguishers, etc.). The mission during the preparedness phase is to monitor the fire and early warning. Monitor the smoke concentration, temperature, and ultraviolet light, and use the incident filtering system to set filtering parameters. As long as all three data reach the alarm range, it is a fire disaster. Contact the surrounding fire department and issue early warning messages to the residents. During the emergency response phase, using the position of the alarm sensors, determine the fire range and spreading speed. Monitoring the surrounding traffic conditions in real time, and work out the police route based on the shortest path model. In the post-disaster recovery phase, social statistical data is used to calculate economic losses and population casualties, and the fire area is calculated based on the area of the fire building.

Table 1. Observation missions, data and methods in four phases

\begin{tabular}{|c|c|c|c|}
\hline Stage & $\begin{array}{l}\text { Observation } \\
\text { mission }\end{array}$ & Observation Data & Observation method \\
\hline Mitigation & $\begin{array}{l}\text { Collecting data relate } \\
\text { to urban fire } \\
\text { cases }\end{array}$ & $\begin{array}{l}\text { Basic geographic data } \\
\text { Location of fire stations } \\
\text { Location of hospitals } \\
\text { Location of emergency } \\
\text { resource }\end{array}$ & $\begin{array}{l}\text { Ground survey(main), Satellite remote } \\
\text { sensing, Aerial survey } \\
\text { Satellite remote sensing(main), Ground } \\
\text { survey, Aerial survey } \\
\text { Satellite remote sensing(main), Ground } \\
\text { survey, Aerial survey } \\
\text { Ground survey(main), Satellite remote } \\
\text { sensing, Aerial survey }\end{array}$ \\
\hline Preparedness & $\begin{array}{l}\text { Fire monitoring and } \\
\text { early warning }\end{array}$ & $\begin{array}{l}\text { Meteorological data } \\
\text { Smoke concentration data } \\
\text { Temperature data } \\
\text { UV spectral data }\end{array}$ & $\begin{array}{l}\text { In-situ sensor (main, encrypted } \\
\text { observation), satellite remote sensing } \\
\text { Smoke sensor } \\
\text { Temperature sensor } \\
\text { UV spectral sensor }\end{array}$ \\
\hline Response & $\begin{array}{c}\text { Emergency } \\
\text { monitoring and } \\
\text { disaster assessment }\end{array}$ & $\begin{array}{c}\text { Meteorological data } \\
\text { Smoke concentration data } \\
\text { Temperature data } \\
\text { UV spectral data } \\
\text { Transportation data }\end{array}$ & $\begin{array}{c}\text { In-situ sensor (main, encrypted } \\
\text { observation), satellite remote sensing } \\
\text { Smoke sensor } \\
\text { Temperature sensor } \\
\text { UV spectral sensor } \\
\text { Cameras(main), Satellite remote } \\
\text { sensing }\end{array}$ \\
\hline
\end{tabular}

Casualty data

Recovery Loss assessment Socioeconomic data

Burned area data
Manual statistical survey

Manual statistical survey

Satellite remote sensing(main), Ground survey, Aerial survey

\section{URBAN FIRE INCIDENT MODELING SYSTEM}

\subsection{System Design}

There is no standardized system for modelling fire incident currently, so this paper designed an urban fire incident management system which is a unified management and service platform for urban fire incidents. This system has urban fire event modeling, event query, and event management functions. Eventually, it can establish and manage an incident library for real-time monitoring of urban fire incidents, issue early warning, emergency response and event assessment and issues early warnings. This system can build a model of urban fire incidents by entering the observation information in four phases, and the model can be managed, saved in the system, and models built through this software can also be used as a description model of intelligent decision results.

\subsubsection{System Implementation}

This system uses a C / S structure, an MVC architecture, and has a data layer, a business layer, and a display layer. The data layer includes geographic data, historical data related to fire incidents, meteorological data and data from three physical sensors, including smoke concentration, temperature, and ultraviolet light. The business layer includes event modeling, query and filtering, and format conversion tools. The display layer includes the interface for modeling, querying, and filtering. Users can enter observation parameters, view, modify, delete models and enter query conditions. 


\subsubsection{System function}

The system mainly has three functions, which are urban fire incident modeling, urban fire incident query, and urban fire incident observation information filtering.

1) Urban fire incident information modeling: By enter urban fire information in four phases, the software will build a modeling template to generate urban fire incident models.

\subsection{Modeling System Implementation}

The urban fire incident management system has three modules, event modeling, event query, and event management. The event modeling module can be used to loading and model generation of urban fire incident models, as shown in the Figure 3 below. There are four phases, for each phase the input information is different, after input all the information, by click the confirm

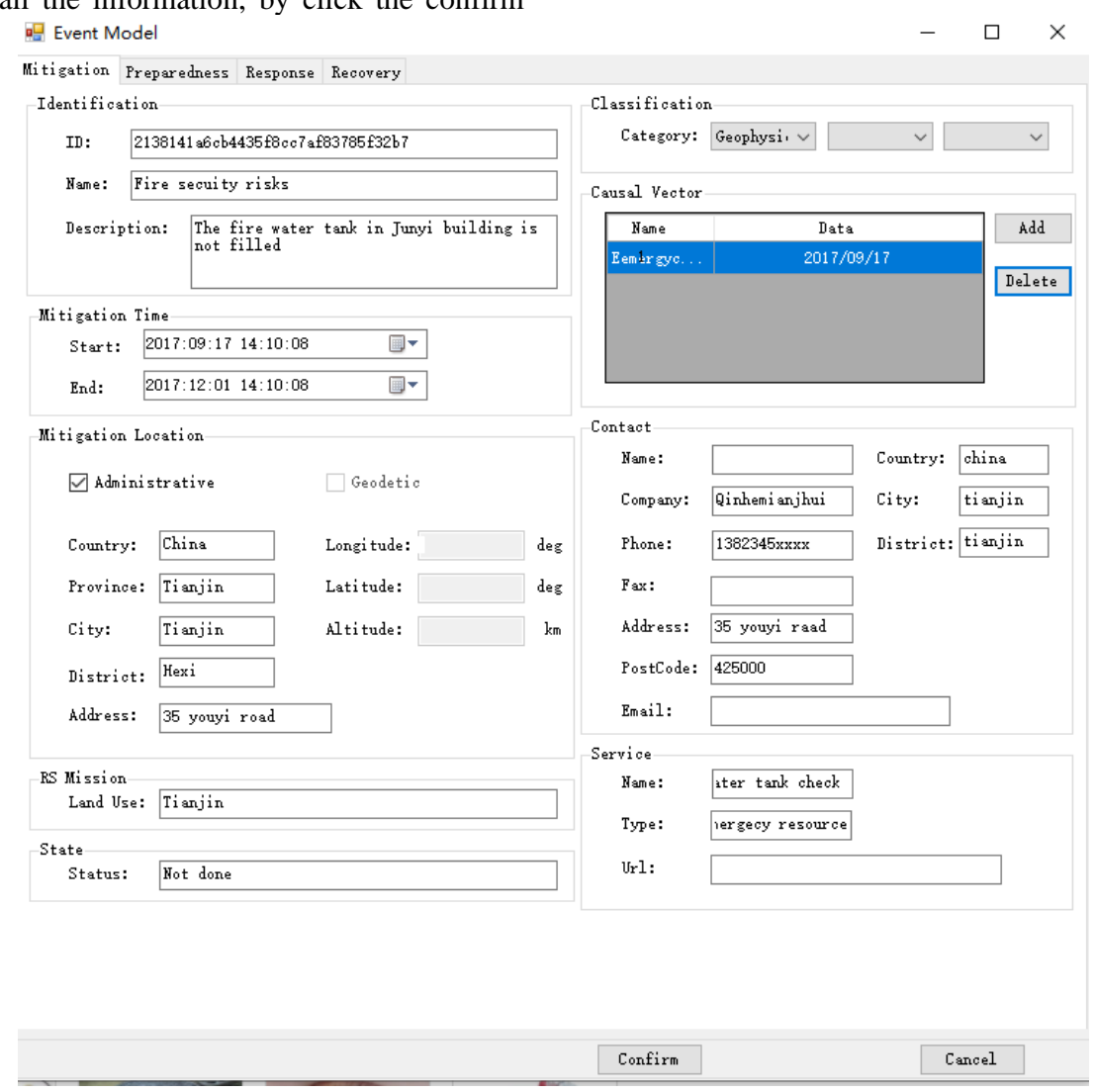

Figure 3. Urban fire event modeling function module and fuzzy query.
2) Urban Fire Incident Query: The existing cases in the system can be queried through the entered query conditions. There are multiple query methods such as simple query, combined query

3) Filtering of urban fire event observation information: Filters set by filtering codes are used to set filtering parameters to filter three types of urban fire event observation information.

button, an instance is established according to the urban fire incident meta-model framework, and then an incident model is generated.

In the event query module as shown in the Figure 4, once a city fire incident is built, it can be saved in the system, and can be queried based on the time, place and category information of the

the incident in the query function, as shown in the figure 4.

In the event management module, users can modify, delete, browse, and filter the existing fire incident models. According to the filtering code, edit the filtering parameters, and set the filtering range of the three-observation data. For example, the smoke concentration exceeds $0.06 \%$, the temperature exceeds 400 degrees Celsius, and the ultraviolet rays between $180 \mathrm{~nm}$ and $260 \mathrm{~nm}$ Alarm, when the three sensors alarm at the same time, it is a fire incident, contact the fire department to issue an alarm.

\section{EXPERIMENT ANALYSIS}

\subsection{Experiment Background}

According to Beijing Youth Daily, on December 1, 2017, a fire broke out on the 38th floor of the city building at the intersection of Youyi Road and Pingjiang Road, Hexi District, Tianjin. 10 people dead and 5 were slightly injured. This is a major fire accident. The direct cause of the fire accident is that the leftover sources ignited the combustible materials stored the fire elevator of the 38th floor in the Junyi Building (Zhang 2018). Four different phases of the fire accident were modeled in the paper and the detail information of the fire were shown in the figure 5 .

\subsection{Experiment Result}

The results of modeling four phases in Tianjin's "12.1" major fire incidents, were shown in Figure 6 and Figure 7. During the 
mitigation phase, emergency resources were checked to handle potential fire hazards. In this example, the fired building was reminded by other companies two months ago that the fire water tank was not filled with water. It was a hidden fire risk, and not

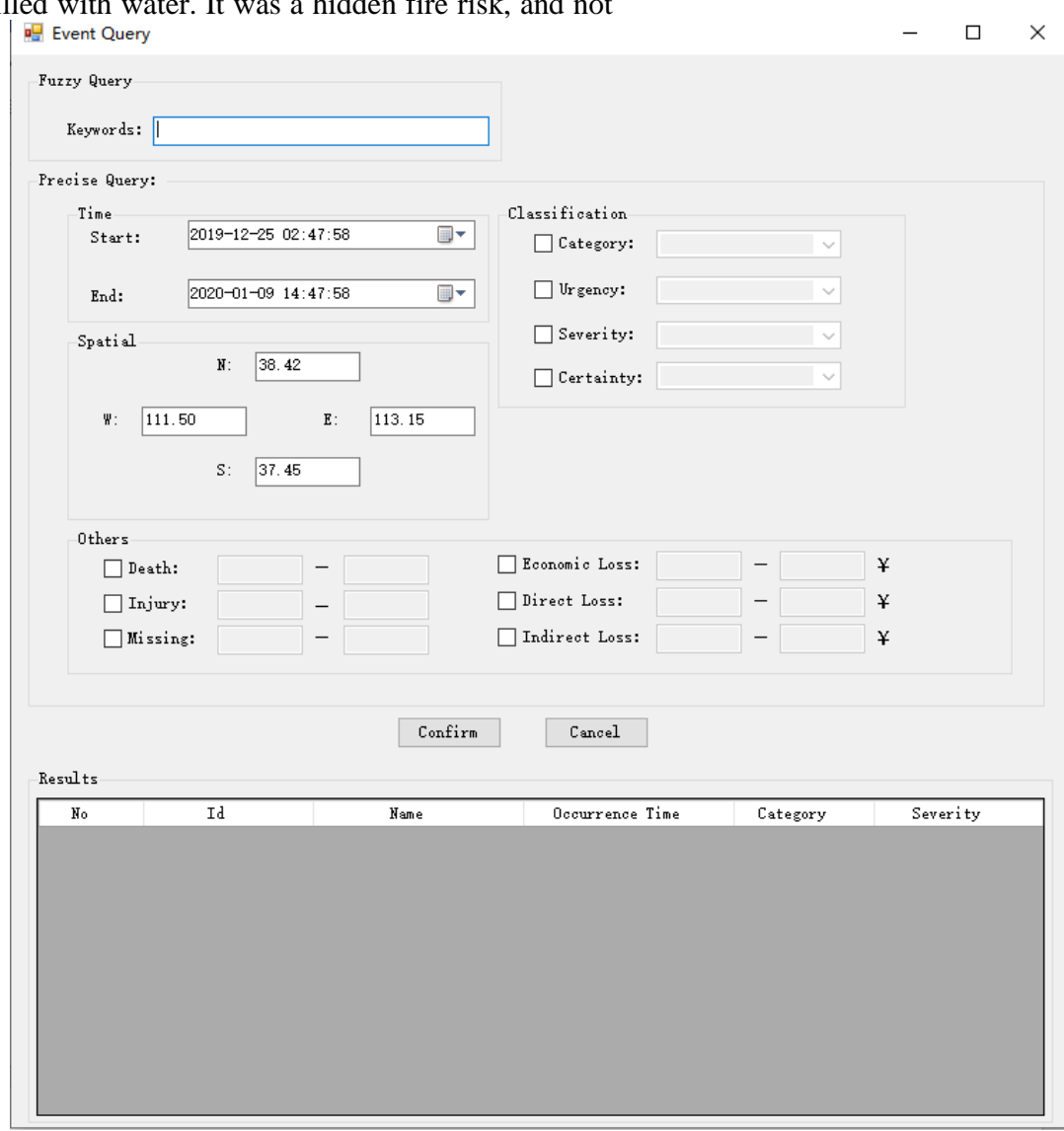

Figure 4. Urban fire event query function module

floors of the building. So that the fire position and the possible range of the fire could be judged by the positions of the smoke sensors. At first, it was the smoke sensor in front of a fire elevator in the $38^{\text {th }}$ floor alarm, the sensors in stairs, elevator, aisle in the $38^{\text {th }}$ floor alarm later, and then the sensors in $39^{\text {th }}$ floor alarm. It means that the fire start at $38^{\text {th }}$ floor's fire enough attention was paid by the managers of the building, eventually the fire occurred. During the preparedness phase, the smoke sensors made multiple alarms in different positions and

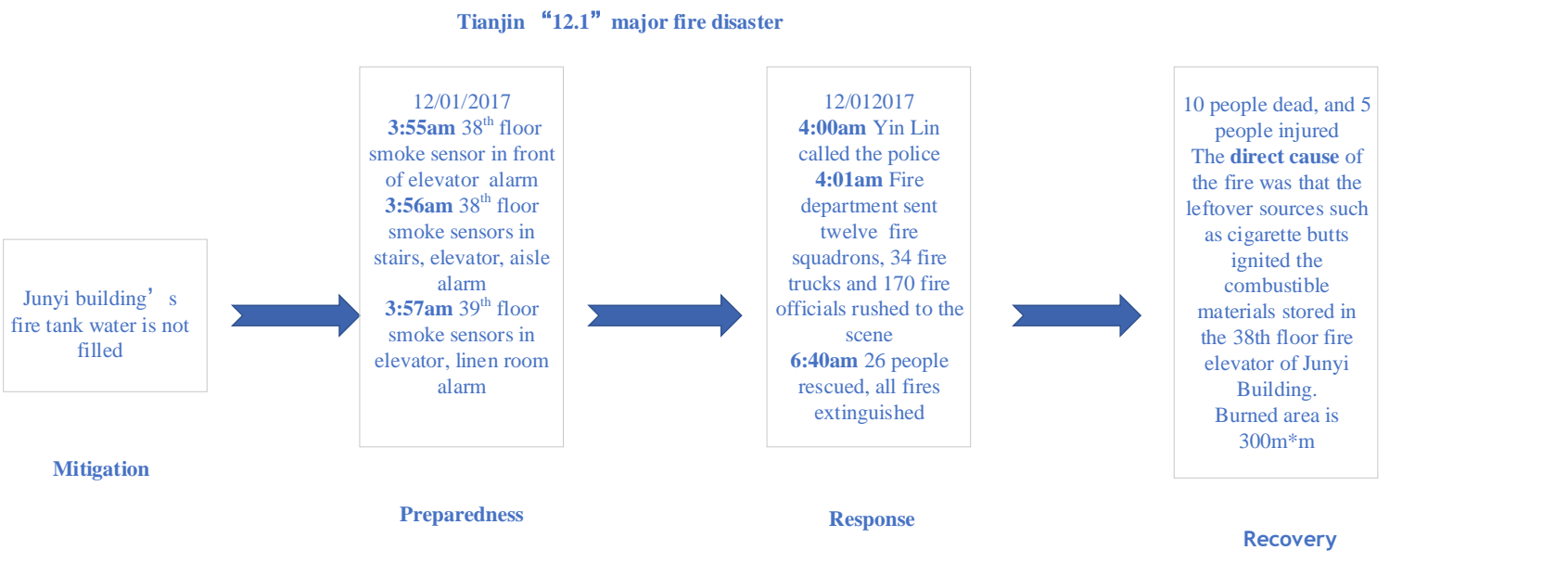

Figure 5. Detail information of Tianjin "12.1" major fire disaster

trucks. After the fire forces arrived at the building, they spend two hours fighting the fire, and rescue 26 people from the elevator, and then it spread to $39^{\text {th }}$ floor. During the emergency response phase, the fire department sent fire forces to the scene to fight the fire. Using the real-time transportation information from the camera in the street, and combined with the shortest path model, the fire department can get a best route for fire 
on real-time data. During the post-disaster recovery phase, social statistics data were used to obtain information on casualties and economic losses. There were 10 people dead, and 5 people injured in this incident. The direct cause of this

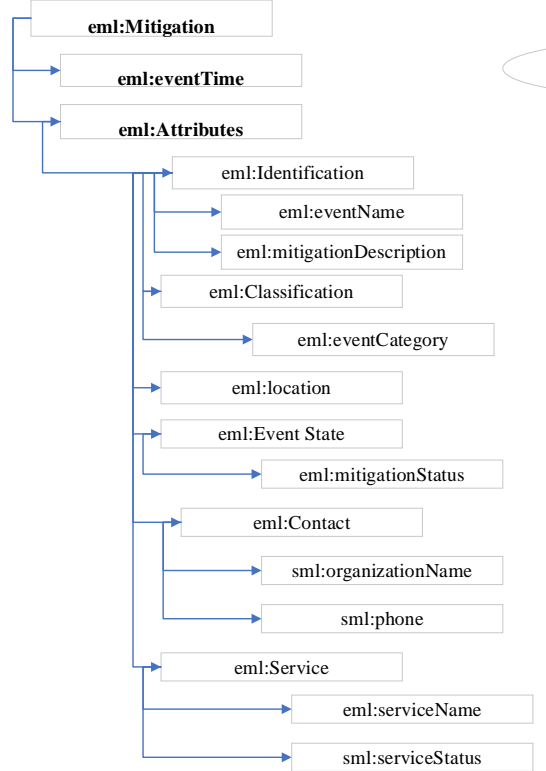

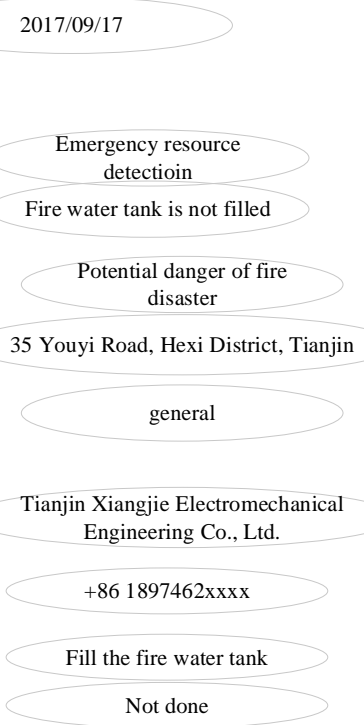

incident is that the leftover sources such as cigarette butts ignited the combustible materials in the $38^{\text {th }}$ floor fire elevator of the building, and the burned area is $300 \mathrm{~m} * \mathrm{~m}$.

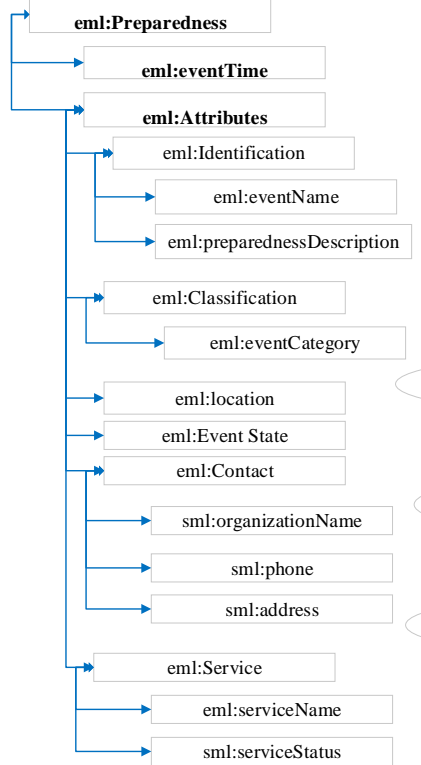

2017/12/01 03:55

Smoke sensor alert

$38^{\text {th }}$ and $39^{\text {th }}$ floor smoke sensor alert

Potential danger of fire disaster

35 Youyi Road, Hexi District, Tianjin Emergency

Tianjin Xiangjie Electromechanical Engineering Co., Ltd.

$+861897462 \times x x x$

35 Youyi Road, Hexi District, Tianjin

Check the smoke sensor in $38^{\text {th }}$ and $39^{\text {th }}$ floor Not done

Figure 6. Mitigation and preparedness phase modelling result

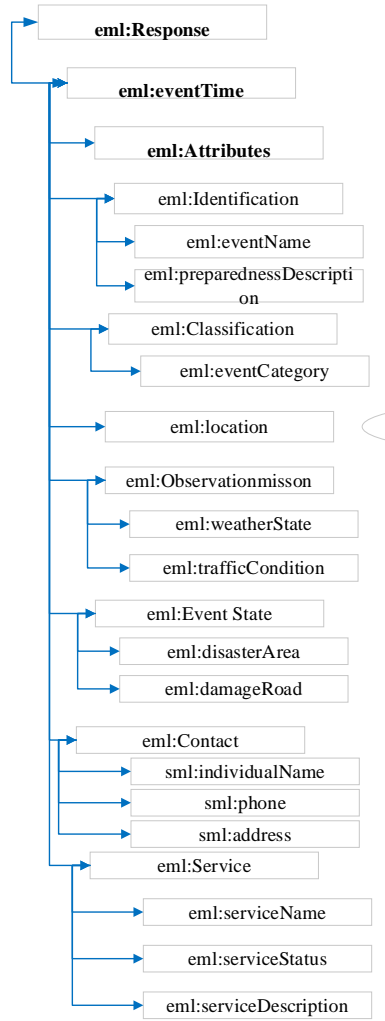

2017/12/01 04:00

Call the police

It is smoky outside

Fire disaster

35 Youyi Road, Hexi District, Tianjin

Temperature and wind direction data

Temperature and wind

direction data

$37^{\text {th }}, 38^{\text {th }}$ and $39^{\text {th }}$ floor of

Junyi building

Youyi Road

liuyin

+86 1593462xxxx

35 Youyi Road, Hexi District, Tianjin

Fire station action Done

Dispatched 12 squadrons, 4 fire truck and more than 170 fire officers to the scene

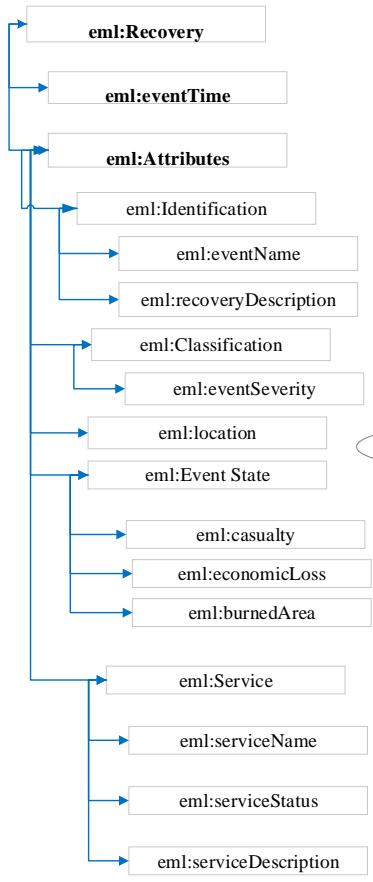

2017/12/03

"12.1" Major Fire Incident in Tianjin

Search and rescue 26 people trapped on the 38th and 39th floors. The fire was all extinguished

Major fire disaster

35 Youyi Road, Hexi District, Tianjin

10 dead, and 5 injured

25160.6 thousand yuan

$300 \mathrm{~m} 2$

Invesgation

Done

Cigarette butts ignite the mbustibles stored in the front of the fire elevator of the 38th floor.

Figure 7. Response and recovery phase modelling result

\section{DISCUSSION AND CONCLUSION}

Discussion 
This paper made a preliminary research on the information description model and modeling method of urban fire incidents. Different from other scholars' research related to fire modeling, this method proposed in this paper can combine with the real time data to promote the evolution of incidents, and the descriptions of the fire incidents are more detail and clear.

On the basis of this analysis and previous work, EML based urban fire incident modeling method is useful in the fields of representing the dynamic information and achieving the dynamic modeling.of urban fire incidents. By modeling urban fire incidents, it can improve the command effectiveness of the

Conclusion

The descriptions of existing fire model are too singular, and there is no unified standardized language to describe a fire incident. This paper proposes an urban fire incident modeling system and an EML-based urban fire incident modeling method. Based on the framework of MOF, different phases of fire incidents were constructed, and real-time observation of the Internet of Things data would be used to monitor the development of fire incidents, judge the scope of the fire development, and help fire departments to formulate a better firefighting plan. The main work of this article includes the following:

1) Concluded different observation tasks, observation data, and observation methods in four phases of a fire incident.

2) Analysed the characteristics of different fire incidents, summarize the requirements for fire incident characterization, and propose a nine-tuple description model for describing fire incidents, including identification information, classification information, archive information, status information, time information, space information, observation information, and contact information, and service information.

3) Developed an urban fire incident modeling software, and it has three functions. Modeling the fire incidents, save the template to the system and set the filter parameters for the fire incidents.

4) Used EML to model Tianjin's "12.1" major fire events, the feasibility of the proposed dynamic modeling of urban fire events and the effectiveness of the fire event information model were verified.

\section{ACKNOWLEDGEMENTS}

This work was supported by the National Key Research and Development Program of China(2018YFB2100603).

\section{REFERENCES}

Everding T, Echterhoff J. Evernt Pattern Markup Language (Version 0.3.0) Open Geospatial Consortium, Inc. OGC Document Number: OGC 08-132, 2008 commanders at the scene of the fire incidents, and assist them in formulating the best rescue plan. Besides, it can also provide evidence for the investigation of the cause of the fire.

However, it should be noted that this study has used only historical data to build the model of fire incidents, and the results are lack of real time observation data. It is not clear that if this method can be equally effective in real time cases, and it has not been examined in this paper. An experiment that can collect the real time observation data can improve this, which should be the main work in the future.

Haddow G, Bullock J. Introduction to emergency management $[\mathrm{M}]$. Amsterdam: Butterworth-Heinemann, 2003

ISO/IECC 19502. Information Technology-Meta Object Facility (MOF). International Standard Organization, 2005

Liu Jing. Modeling of firefighting process based on Petri nets [C]. // Chinese Science and Technology Association. 2006 Chinese Association of Science and Technology. 2006

National Bureau of Statistics of the People's Republic of China. China Statistical Yearbook [M]. Beijing: China Statistics Press, 2019

OMG’s MetaObject Facility, http://ww.omg.org/mof, 2014

Xie Meiyu. Research on Building Fire Emergency Decision Model Based on CBR and Bayesian Networks [D].2018

Zhu Yiping, Fan Weicheng, Xue Haisheng, et al. A decision method for urban firefighting dispatch based on GIS and Petri nets [J]. Journal of University of Science and Technology of China,2005

Zhong Kaibin. (2009). "One case, three systems": the basic framework for the construction of China's emergency management system. Nanjing Social Sciences (11), 83-89.

Zhang ya, 2018. Tianjin "12.1" fire disaster, Beijing Youth Daily, July 3, 2018 